\title{
Polymer-coated pH-responsive high-density lipoproteins
}

Hyungjin Kim ${ }^{\text {a }}$, Haruki Okamoto ${ }^{\text {a }}$, Arnaud E. Felber ${ }^{\text {b }}$, Anna Polomska ${ }^{\text {b }}$, Nobuhiro

Morone $^{\text {a }}$, John E. Heuser ${ }^{\text {a }}$ Jean-Christophe Leroux ${ }^{b}$, Tatsuya Murakami ${ }^{\text {a,* }}$

${ }^{a}$ Institute for Integrated Cell-Material Science (WPI-iCeMS), Kyoto University,

Sakyo-ku, Kyoto 606-8501, Japan.

${ }^{b}$ Department of Chemistry and Applied Biosciences, Institute of Pharmaceutical

Sciences, ETH Zürich, Vladimir-Prelog-Weg 1-5/10, 8093 Zurich, Switzerland.

* Corresponding Author,

E-mail address: murakami@icems.kyoto-u.ac.jp 


\begin{abstract}
Intracellular drug delivery by nanoparticles is often hampered by their endosomal entrapment followed by their degradation in the lysosomal compartment and/or exocytosis. Here, we show that internalization and endosomal escape of cargoes in a cationized natural nanocarrier, high-density lipoprotein (HDL), can be controlled in a pH-dependent manner through stable complexation with a membranolytic anionic block polymer. A genetically and chemically cationized form of HDL (catHDL) is prepared for the first time by both genetic fusion with YGRKKRRQRRR peptide and incorporation of 1,2-dioleoyloxy-3-(trimethylammonium)propane. Upon addition of poly(ethylene glycol)-block-poly(propyl methacrylate-co-methacrylic acid) (PA), catHDL yields inhibition of internalization at neutral $\mathrm{pH}$ and its subsequent recovery at mildly acidic $\mathrm{pH}$. catHDL forms a stable discoidal-shape complex with PA (catHDL/PA) (ca. $50 \mathrm{~nm}$ in diameter), even in the presence of serum. Significant enhancement of endosomal escape of a catHDL component is observed after a 1-h treatment of human cancer cells with catHDL/PA. Doxorubicin and curcumin, fluorescent anti-cancer drugs, encapsulated into catHDL/PA are also translocated outside of endosomes, compared with that into catHDL, and their cytotoxicities are enhanced inside the cells. These data suggest that catHDL/PA may have a potential benefit to improve the cellular delivery and endosomal escape of therapeutics under mildly acidic conditions such as in tumor tissues.
\end{abstract}

\title{
Keywords:
}

endosomal escape, internalization, membranolytic polymer, anticancer drug delivery, mildly acidic $\mathrm{pH}$ 


\section{Introduction}

Despite continuous advances in nanoparticulate delivery systems, the efficient and specific delivery of therapeutic drugs to desired cellular compartments is often limited by their intracellular trafficking pathway. The endocytic pathway is the major cell uptake mechanism for therapeutic nanoparticles. Nanoparticles are generally taken up by various forms of endocytosis and are then rapidly trafficked to the lysosomes that contain a range of hydrolytic enzymes $[1,2]$. In the lysosomes, nanoparticles and their content can be degraded or alternatively other trafficking pathway can lead to their exocytosis [3]. In both cases, the outcome is a loss of the pharmacological activity. Thus, the endosomal escape of nanoparticles (or of their cargo) may help improving the intracellular bioavailability of drugs and increase their therapeutic efficacy [4]. Here, we sought to develop a nanocarrier rationally designed to deliver its cargoes outside the endo/lysosomes.

Generally, nanoparticles that are internalized via endocytic pathways rapidly ( $<2 \mathrm{~min}$ ) experience a drop in $\mathrm{pH}$ to $c a .5 .9-6.0$ in the lumen of early/recycling vesicles, and further to $c a .5 .0$ during transport from the late endosomes to lysosomes [5]. Such mildly acidic conditions have been used to trigger the endosomal escape of therapeutic compounds loaded into nanoparticles [6]. Utilization of polyanions bearing both pendant carboxylate groups and alkyl chains is one of such examples. Following $\mathrm{pH}$ decrease, they dynamically change their conformation from an expanded hydrophilic coil (neutral and basic $\mathrm{pH}$ ) to a hydrophobic globule (acidic $\mathrm{pH}$ ), and this transition can be exploited to destabilize transiently endosomal membranes $[7,8]$. Meanwhile, extracellular $\mathrm{pH}$ in normal tissues is kept constant at $\mathrm{pH} 7.2-7.6$, while 
tumor $\mathrm{pH}$ is typically 0.4 units lower [9]. Therefore, low $\mathrm{pH}$-dependent intracellular drug delivery could also be a smart strategy in cancer chemotherapy.

High-density lipoprotein (HDL) is a natural nanoparticle of approximately 10 $\mathrm{nm}$ in diameter in the body and its discoidal form (nascent HDL) is easily reconstituted with recombinant apolipoprotein A-I protein (apoA-I) and phospholipids. Recently, HDL has received increasing attention for use as a drug carrier because of its innate biocompatibility, its small size favorable for deep tissue penetration, the presence of the lipid core suitable for drug loading, and the wide variety of possible functionalization (genetic and chemical modification of apoA-I and chemical modification of the lipid bilayer) $[10,11]$. We have developed a highly-internalizable HDL composed of genetically modified apoA-I with TAT peptide, derived from HIV Tat protein, and 1-palmitoyl-2-oleoyl-sn-glycero-3-phosphocholine (POPC) [12], and reported its utility as a drug carrier [13] as well as a cell biology research tool [14]. This HDL mutant was able to potentiate the pharmacological action of DXR in vitro and in vivo, compared to wild-type HDL. This potentiation could be attributed to a better intracellular delivery mechanism. Nevertheless, the majority of DXR delivered by the HDL mutant remained entrapped in the endosomes. This motivated us to develop an improved HDL mutant capable of efficiently escaping from the endosomal compartment.

In this study, we sought to prepare stable complexes between the HDL mutant with TAT peptide and poly(ethylene glycol)-block-poly(propyl methacrylate- $c o$-methacrylic acid), $\mathrm{PEG}_{115}-b-\mathrm{P}\left(\mathrm{PrMA}_{22}-c o-\mathrm{MAA}_{52}\right)[15,16](\mathrm{PA})$, to confer mildly acidic $\mathrm{pH}$-dependent internalization and membranolytic abilities to HDL. To enhance accommodation of PA, the HDL mutant was further cationized with 1,2-dioleoyloxy-3-(trimethylammonium)propane (DOTAP) or dimethylaminoethane 
carbamoyl cholesterol (DC-chol). Complexation of these cationized HDL mutants and PA was optimized, and the structure and in vitro function of the HDL-PA complexes were investigated in detail.

\section{Material and methods}

\subsection{Materials}

POPC was from NOF (Tokyo, Japan). DOTAP and dimethylaminoethane carbamoyl cholesterol (DC-chol) were purchased from Avanti Polar Lipids (Alabaster, AL, USA). Doxorubicin hydrochloride (DXR), agarose, and SDS-PAGE precast gel were obtained from Wako (Osaka, Japan). Curcumin (CCM) and Bafilomycin A1 were from Sigma-Aldrich (St. Louis, MO, USA). Tetramethylrhodamine isothiocyanate (TRITC) were from Fluka (St. Gallen, Switzerland). 5-(Aminoacetamido)fluorescein, Alexa Fluor 546 succinimidyl ester (Alexa546) and LysoTracker Green were from Molecular Probes, Inc. (Eugene, OR, USA). AcidiFluor ORANGE were from Goryo Chemical, Inc. (Sapporo, Japan). Dc Protein Assay Kit was from Bio-Rad Laboratories (Hercules, CA, USA). Spectra/Por Dialysis Membranes (molecular weight cut-off (MWCO), 3500 and 50,000) were obtained from Spectrum Laboratories (Rancho Dominguez, CA, USA). Cell Counting Kit-8 and WSC (water soluble carbodiimide) were obtained from Dojindo Laboratories (Kumamoto, Japan). NAP-5 Column was purchased from GE Healthcare UK Ltd (Buckinghamshire, UK). Fetal bovine serum (FBS) was obtained from Japan Bioserum (Hiroshima, Japan). Dulbecco's PBS and trypsin-EDTA (0.05\% trypsin, $0.53 \mathrm{mM}$ EDTA-4Na) were obtained from Invitrogen 
(Carlsbad, CA, USA). DMEM and RPMI1640 media were purchased from Wako. Cell culture dishes were from BD Bioscience (San Jose, CA, USA). Glass bottom dishes were from IWAKI (Tokyo, Japan). E. coli strain BL21(DE3) was from Novagen (Madison, WI, USA). Ni Sepharose was purchased from GE Healthcare Life Sciences (Little Chalfont, Buckinghamshire, UK).

\subsection{Preparation of PA-cationized HDL mutant complexes}

Synthesis of poly(ethylene glycol)-block-poly(propyl

methacrylate-co-methacrylic acid) (PA) composition: $\mathrm{PEG}_{115}-b-\mathrm{P}\left(\mathrm{PrMA}_{22}-c o-\mathrm{MAA}_{52}\right)$ was described elsewhere [16]. Cationized HDLs were reconstituted according to the previous report with minor modifications [12]. Briefly, the required amounts of a chloroform-methanol solution of POPC, and DOTAP or DC-chol were mixed in a round-bottomed glass flask. The organic solvent was removed using an evaporator and then under vacuum overnight. The dried lipid mixture was solubilized in PBS containing $30 \mathrm{mg} / \mathrm{mL}$ sodium cholate at a molar ratio of lipid:cholate of $1: 4$. The TAT peptide-fused apoA-I mutant in PBS containing 4M urea and the mixed lipid were mixed at a lipid/protein molar ratio of 100 [17]. The mixture was incubated overnight at room temperature and then dialyzed against PBS at $4{ }^{\circ} \mathrm{C}$ with a Spectra/Por Dialysis Membrane (MWCO 50,000). The reconstituted cationized HDL mutant solution was centrifuged at $15,300 \mathrm{~g}$ at $4^{\circ} \mathrm{C}$ for $30 \mathrm{~min}$ to remove any debris. Complexation of the cationized HDL mutants and PA was done by simply mixing them in PBS (pH 7.4) at an indicated PA/lipid weight ratio at room temperature for $10 \mathrm{~min}$. PA-cationized HDL mutant complexes were diluted with neutral ( $\mathrm{pH}$ 7.4) or acidic media ( $\mathrm{pH}$ 5.5-6.0) and 
then incubated at room temperature for $15 \mathrm{~min}$, prior to any analyses. For cell studies on $\mathrm{pH}$ dependency, a serum-free DMEM at $\mathrm{pH} 7.4$ or 5.5-6.0 was used, and in the case of long-term treatment $(>1 \mathrm{~h})$ at neutral $\mathrm{pH}, 10 \%$ FBS was added.

\subsection{Agarose gel mobility shift assay}

Agarose gel (0.8\%) was prepared by dissolving agarose powder in $50 \mathrm{mM}$ HEPES (pH 7.4) or $50 \mathrm{mM}$ MES (pH 5.6). Then, gel electrophoresis was performed in each buffer solution to analyze catHDL/PA (4 $\mu \mathrm{g}$ protein/lane). After electrophoresis, the agarose gel was subjected to Coomassie Brilliant Blue G-250 (CBB) staining (Nacalai tesque, Kyoto, Japan) to visualize the protein moiety of the complexes. To investigate the stability of catHDL/PA in the presence of serum by agarose gel electrophoresis, catHDL was labeled with amine reactive Alexa546. The Alexa546-labeled complex (Alexa546-catHDL/PA) was incubated in the presence of $10 \% \mathrm{FBS}$ for $1 \mathrm{~h}$ at $37^{\circ} \mathrm{C}$, and then electrophoresed.

To visualize PA in the agarose gel, fluorescently labeled-PA was synthesized as describe below. $900 \mu \mathrm{g}$ of PA ( $80 \mathrm{nmol}$, or 4.2 unit $\mu \mathrm{mol}$ of $-\mathrm{COOH})$ was reacted with $32 \mu \mathrm{g}$ of 5-(aminoacetamide)fluorescein $(80 \mathrm{nmol})$ in the presence of $1.6 \mathrm{mg}$ of WSC $(8.4 \mu \mathrm{mol})$ at room temperature for $4 \mathrm{~h}$ in water. This equal molar ratio of PA and the fluorescein was selected to minimize the effects of the labeling on the complexation. The fluorescein-labeled PA was purified by NAP-5 column equilibrated with deionized water. The fluorescence images of the agarose gels were obtained using a BIO-RAD ChemiDoc XRS Plus imager (Hercules, CA, USA). 


\subsection{TEM measurement}

For fine structural analysis of catHDL/PA, the mica flake TEM technique was utilized. One drop of a suspension of finely ground muscovite mica flakes were added to $30 \mu \mathrm{L}$ of catHDL/PA suspension at $10-100 \mu \mathrm{g} / \mathrm{mL}$, and catHDL/PA was allowed to gently adsorb to mica for $\sim 30 \mathrm{~s}$ at room temperature, whose procedures were done under pH7.4 or 5.6. The rapidly frozen slurry of mica flakes using liquid helium (Variant Instruments, MO) was then fractured at $-104^{\circ} \mathrm{C}$ in JEOL EM-1950 JFDII freezing unit and immediately deep-etched for $2 \mathrm{~min}$ at $-104^{\circ} \mathrm{C}$ (Tokyo, Japan). It was rotary-shadowed with platinum/carbon at an angle of $11^{\circ}$ from the surface and with carbon from the top. The replica was separated from the mica, cleaned on hydrofluoric acid overnight, washed with water, and picked up on the Formvar-coated TEM grids. The replica was observed in a JEOL JEM1400 at $80 \mathrm{kV}$ (Tokyo, Japan). The TEM images (anaglyphs) were generated from tilted images at $\pm 10^{\circ}$. Use the red and green glasses to see the images as the anaglyphs (red glass on your left).

\subsection{Incorporation of anticancer drugs into catHDL}

Incorporation of DXR into catHDL was performed by incubation of 100 $\mu \mathrm{g} / \mathrm{mL}$ catHDL on a protein basis with $400 \mu \mathrm{g} / \mathrm{mL}$ DXR at $50^{\circ} \mathrm{C}$ for $30 \mathrm{~min}$ [18]. Purification was performed with a NAP-5 column equilibrated with phenol red-free RPMI1640 to remove free DXR and then by centrifugation at $15,300 g$ at $4^{\circ} \mathrm{C}$ for 10 min to remove any debris. Complexation with PA was done in the same manner as described above. DXR concentration in the complex (DXR@catHDL/PA) was 
determined spectrophotometrically at $485 \mathrm{~nm}$ in $2 \%$ sodium dodecyl sulfate to be 3.2 $\mu \mathrm{g} / \mathrm{mL}$. In case of CCM, however, it was encapsulated by different method because of its poor solubility in water. CCM, POPC, and DOTAP were dissolved in chloroform at a drug/lipid weight ratio of 1:10 and the mixture was dried as described above. With this lipid film containing CCM, catHDL was prepared. The CCM concentration was determined spectrophotometrically at $425 \mathrm{~nm}$ in $2 \%$ sodium dodecyl sulfate to be 212 $\mu \mathrm{g} / \mathrm{mL}$.

2.6. In vitro release of $D X R$ from $D X R @$ cat $H D L$

DXR@catHDL or the DOTAP-free formulation (DXR@HDL) $(2.5 \mu \mathrm{g} / \mathrm{mL}$ DXR, $3.5 \mathrm{~mL}$ ) was dialyzed (MWCO 50,000) against $0.9 \% \mathrm{NaCl}(50 \mathrm{~mL})$ containing $0.1 \mathrm{w} / \mathrm{w} \%$ bovine serum albumin in a centrifuge tube with mildly rotating at $37^{\circ} \mathrm{C}$ [12]. DXR in the dialysate was analyzed by a spectrofluorometer FluoroMax-4 (HORIBA JOBIN YVON, Kyoto, Japan) with excitation/emission at 467/555 nm.

\subsection{Cell culture}

T24 human urinary bladder cancer cells was from ATCC (Manassas, VA, USA) and maintained in McCoy's 5A medium supplemented with 10\% FBS. For cell studies, the cells were cultured in DMEM or phenol red-free RPMI1640 medium supplemented with $10 \%$ FBS. All cell culture media contained $100 \mathrm{U} / \mathrm{mL}$ penicillin and $100 \mu \mathrm{g} / \mathrm{mL}$ streptomycin. In some cases, the cells were incubated in the serum-free 
media. T24 cells were incubated at $37^{\circ} \mathrm{C}$ under a humidified atmosphere of $5 \% \mathrm{CO}_{2}$ and 95\% air. Cells were passaged every 3-4 days.

\subsection{Confocal microscopy measurement}

catHDL was reacted with tetramethylrhodamine isothiocyanate (TRITC) at a weight ratio of protein:TRITC of $100: 1$ in $0.1 \mathrm{M} \mathrm{NaHCO}_{3}$ at $4{ }^{\circ} \mathrm{C}$ overnight [19]. Then, excess TRITC was removed by a NAP-5 column equilibrated with PBS, followed by dialysis against PBS. The cells were seeded at $1.0 \times 10^{5}$ cells $/ \mathrm{mL}$ and cultured for 1 day. The cells were treated with $20 \mu \mathrm{g} / \mathrm{mL}$ TRITC-labeled complexes on a protein basis or $3.2 \mu \mathrm{g} / \mathrm{mL}$ DXR@catHDL/PA on a DXR basis. For a mechanistic study, cells were co-treated with $10 \mathrm{nM}$ bafilomycin A1 (Sigma-Aldrich), an endosomal acidification inhibitor, and TRITC-labeled complexes for $1 \mathrm{~h}$ in the presence of serum. The late endosomes/lysosomes of the cells were stained with LysoTracker Green according to the manufacturer's protocol, prior to confocal measurement. Confocal images were acquired using an OLYMPUS FV-10i-LIV confocal laser scanning microscope (Tokyo, Japan). Time-dependent colocalization of their fluorescence was quantitatively analyzed according to the methods previously reported [20] and expressed as colocalization ratio as follows:

Colocalization ratio $=$ TRITC, DXR, or CCM pixels colocalizing with LysoTracker Green or AcidiFluor ORANGE pixels in the cells (yellow in Figs. 4 and 6, and supplementary Figs. 8, 10, 12 and 13) / all TRITC, DXR, or CCM pixels in the cells (yellow + red or yellow + green). TRITC, DXR, or CCM pixels from 3 independent images showing ca. 12 cells were used for colocalization analysis. 


\subsection{Fluorescence-activated cell sorter (FACS) measurement}

The cells $\left(5 \times 10^{4}\right.$ cells $\left./ \mathrm{mL}\right)$ were seeded in 24 -well plate. After one day, the cells were treated with the $1.6 \mu \mathrm{g} / \mathrm{mL}$ DXR or $20 \mu \mathrm{g} / \mathrm{mL}$ CCM-containing samples for $18 \mathrm{~h}(\mathrm{DXR})$ or $5 \mathrm{~h}(\mathrm{CCM})$ at $37^{\circ} \mathrm{C}$ in the presence of $10 \% \mathrm{FBS}$. After washing with PBS twice, the cells were harvested by trypsin treatment. To further eliminate the cell surface-bound DXR@catHDL, the cells were washed with the cell culture medium twice. Finally, the cells were dispersed in PBS containing 2\% FBS and analyzed by a FACS AriaII flow cytometer (Becton Dickinson, NJ, USA). The non-treated cells were used to determine the auto-fluorescence. 3,000 cells were counted for evaluation. Assays were performed in triplicate.

\subsection{Cytotoxicity assay}

The cells $\left(5 \times 10^{4}\right.$ cells $\left./ \mathrm{mL}\right)$ were seeded in 96 -well plate. After one day, the cells were treated with the DXR $(0.8,1.6 \mu \mathrm{g} / \mathrm{mL}$ DXR $)$ or CCM $(20 \mu \mathrm{g} / \mathrm{mL}$ $\mathrm{CCM}$ )-containing samples for $18 \mathrm{~h}$ at $37^{\circ} \mathrm{C}$ in the presence of $10 \% \mathrm{FBS}$. After washing, cell viabilities were evaluated using a Cell Counting Kit-8 and a SpectraMax M2\&M2e Microplate Reader (Molecular Devices). Cell viabilities were normalized to (OD450OD620) for the untreated cells. Assays were performed in triplicate.

\subsection{Statistical analysis}


Statistical differences between two groups were analyzed by using Student t-test. Multiple comparisons for more than two groups were performed by using one-way analysis of variance (ANOVA) followed by Fisher's test to determine the significance of difference among all paired combinations. P-values lower than 0.05 were considered to be statistically significant.

\section{Results and Discussion}

\subsection{Preparation of cationized HDL mutants}

The HDL mutants with varying degree of cationization were prepared by mixing the TAT peptide-fused protein and a mixed lipid of the cationic lipids, DOTAP or DC-chol, and POPC at a cationic lipid/POPC molar ratio of $0-30 \%$. Then, the effect of PA addition on internalization of the cationized HDL mutants was evaluated by confocal microscopy. PA was simply mixed with the cationized HDL mutants at $\mathrm{pH} 7.4$ at a PA/lipid weight ratio of 0.03 (vide infra) [21], and the mixture was incubated for $10 \mathrm{~min}$ at room temperature. Then, a part of the mixture was diluted with neutral or mildly acidic serum-free medium. Human cancer cells (T24) were treated with each of the media at $37^{\circ} \mathrm{C}$ for $1 \mathrm{~h}$. As shown in Fig. 1, internalization of the cationized HDL mutants prepared with $0 \%$ and $10 \%$ DOTAP was suppressed in the presence of PA at $\mathrm{pH} 7.4$, and then recovered at $\mathrm{pH}$ 5.5-6.0. This $\mathrm{pH}$-dependency of the uptake was quantitatively analyzed with fluorescence-activated cell sorter (FACS) (Supplementary Fig. 1), indicating that the effects of PA addition were the most prominent for the mutant prepared with 10 mol\% DOTAP. In addition, this low pH-dependent 
internalization caused no detectable plasma membrane damage, despite the mildly acidic pH-dependent membranolytic ability of PA (Supplementary Fig. 2). This might be derived from differences in the overall membrane structure between the plasma membrane and endosomal membrane, e.g., the lower curvature and higher cholesterol content of the former than the latter [22]. The uptake recovery at low $\mathrm{pH}$, seemed to be less obvious at $20 \mathrm{~mol} \%$ and disappeared at $30 \mathrm{~mol} \%$. This might be accounted for by the relatively low stability of the mutant, because the yield of HDL mutant formation (protein basis) was drastically decreased in the preparation at more than 20 mol\%, while the mean size was comparable among them (Supplementary Table 1). In addition, the cationized HDLs themselves might be further destabilized by the coil-to-globule transition of PA at low $\mathrm{pH}$ to some internalization-impaired forms (vide infra). On the other hand, in the case of DC-chol, such pH-dependency was not obvious at all DC-chol/total lipid ratios (Supplementary Fig. 3). This may be explained by difference in degree of ionization of the amine group at $\mathrm{pH} 7.4$ between DOTAP (100\% charged) and DC-chol (50\% charged) as previously reported by Zuidam et al [23]. Thus, this lower $\mathrm{pH}$-dependency observed for DC-chol would be related to weaker electrostatic interactions between PA and the cationized HDL mutants. Hereafter, the HDL mutant cationized with $10 \%$ DOTAP was used for further evaluation and referred to as catHDL. 


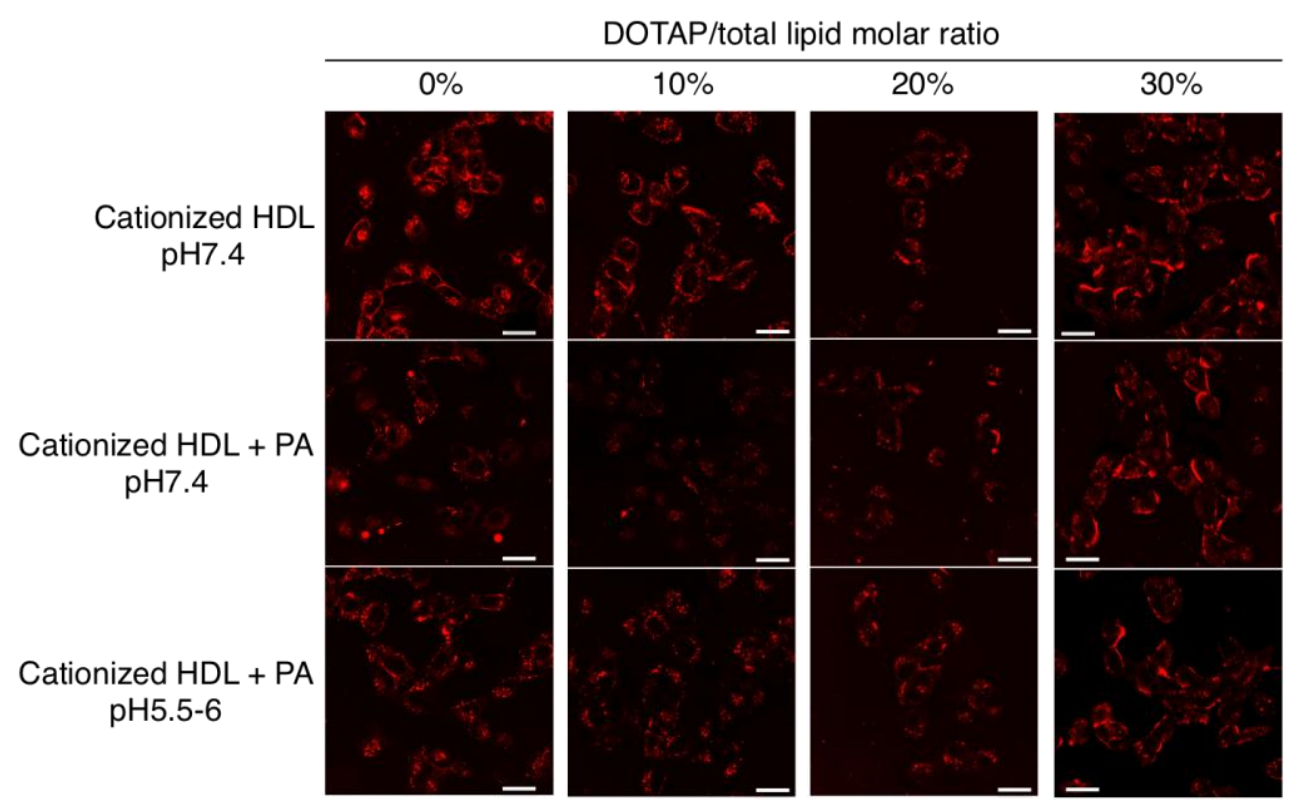

Fig. 1. Low pH-dependent internalization of high-density lipoprotein (HDL). HDL was cationized via both genetic fusion of apoA-I protein with a cationic peptide (YGRKKRRQRRR) and incorporation of a cationic lipid (1,2-dioleoyloxy-3-(trimethylammonium)propane, DOTAP) into the lipid bilayer at a various DOTAP/total lipid molar ratio to facilitate the binding to an anionic polymer, poly(ethylene glycol)-block-poly(propyl methacrylate-co-methacrylic acid) (PA). Four types of the cationized HDL mutant (0-30\% DOTAP) were labeled with tetramethylrhodamine isothiocyanate (TRITC), and then mixed with PA at a PA/lipid weight ratio of 0.03 in PBS ( $\mathrm{pH} 7.4)$. The mixtures were then diluted with neutral $(\mathrm{pH}$ 7.4) or acidic serum-free cell culture medium ( $\mathrm{pH}$ 5.5-6.0). The cells were treated with each of the diluted mixtures for $1 \mathrm{~h}$ at $37^{\circ} \mathrm{C}$, and then analyzed by confocal microscopy. Among the cationized HDLs, the HDL with 10 mol\% DOTAP (catHDL) appears to show a clearest $\mathrm{pH}$-dependency in internalization in the presence of PA. Scale bar, 30 $\mu \mathrm{m}$.

\subsection{Complex formation between catHDL and PA}


To confirm the catHDL-PA complex (catHDL/PA) formation, non-denaturing agarose gel electrophoresis for catHDL mixed with PA at various PA/lipid weight ratios (0-3) at $\mathrm{pH} 7.4$ was performed at both neutral and mildly acidic pHs (Fig. 2a, b). catHDL in the gels was visualized by Coomassie Brilliant Blue (CBB) staining [24]. Any complexes with a pI lower than the buffer $\mathrm{pH}$ carry a net negative charge and migrate toward the anode (lower side of gel). The gel mobility shift of catHDL became apparent at a PA/lipid weight ratio of 0.03 and it appeared to reach a plateau at a ratio of 2.4 at $\mathrm{pH}$ 7.4. At $\mathrm{pH} 5.6$, however, the mobility shift was markedly reduced, and no obvious shift was observed at a ratio of 0.6 or less. These results clearly show the complexation of catHDL and $\mathrm{PA}$ at $\mathrm{pH} 7.4$, and protonation of $\mathrm{PA}$ in the complex at $\mathrm{pH}$ 5.6. The complex formation was also performed using the HDL mutant with no DOTAP (Supplementary Fig. 4). Upon addition of PA, the gel shift was also observed, but the maximal mobility shift was significantly low compared with that for catHDL, indicating that electrostatic interactions play an important role in the complexation. 


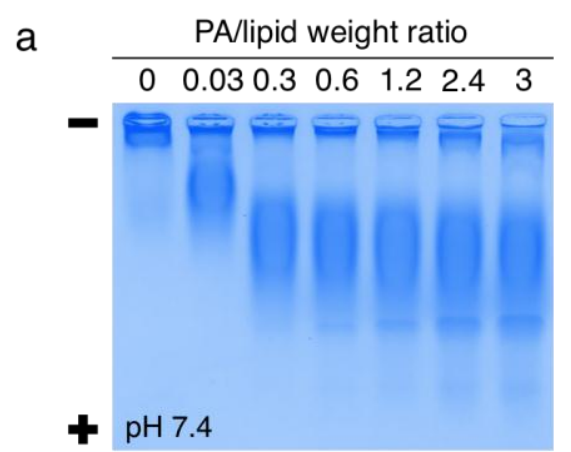

C

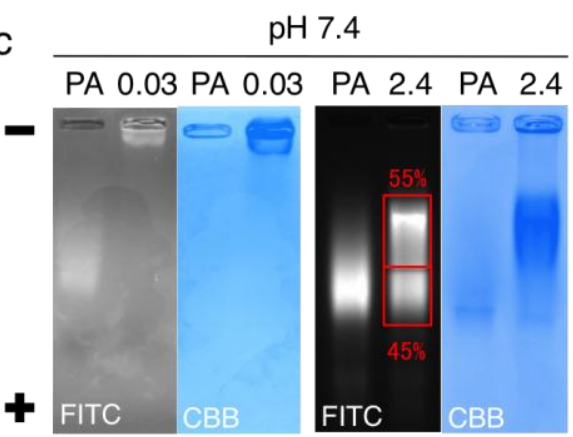

b

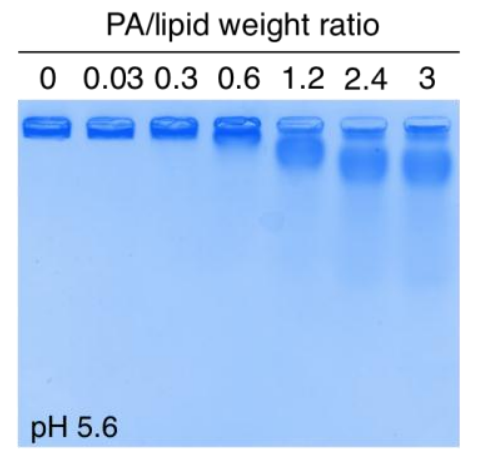

d

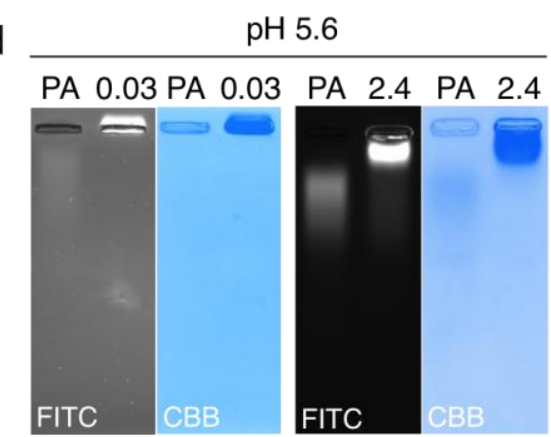

Fig. 2. Complex formation between catHDL and PA. Non-denaturing agarose gel $(0.8 \%)$ mobility shift assay for catHDL/PA was perfomed in the presence of PA at $\mathrm{pH}$ 7.4 (a) or 5.6 (b). After electrophoresis, the protein moiety of the complexes were stained with Coomassie Brilliant Blue (CBB) to detect the apoA-I mutant in the complex. The gel mobility shift of catHDL is apparent at a PA/lipid weight ratio of 0.03 and higher at $\mathrm{pH} 7.4$, whereas significantly suppressed at $\mathrm{pH}$ 5.6, demonstrating that PA was bound to catHDL at $\mathrm{pH} 7.4$ and protonated in the complex at $\mathrm{pH}$ 5.6. Then, PA was fluorescently labeled with 5-(aminoacetamido) fluorescein, and complexation was performed at a ratio of 0.03 or 2.4. Each sample was electrophoresed at $\mathrm{pH} 7.4$ (c) and 5.6 (d). After electrophoreses, fluorescence from PA was detected and it was subjected to $\mathrm{CBB}$ staining to confirm the colocalization of PA and protein moiety of catHDL/PA complex. Only PA, the amount of which was the same as that used for the complexation, was also electrophoresed in the left lane in the gels. Numbers above the gel images indicate a PA/lipid weight ratio adopted for the complexation. Numbers in red in (c) indicate the percentage of the band intensity within each square. In (c), the PA fluorescent band is exactly colocalized with the CBB-stained band for catHDL at a ratio of 0.03 , demonstrating that all PA was bound to catHDL. At a ratio of $2.4,55 \%$ of the 
fluorescence intensity in lane is colocalized with the CBB-stained band. Meanwhile, at pH 5.6 in (d), irrespective of the amount of PA, all the fluorescent bands are colocalized with the CBB-stained band, indicating that bound PA at pH 7.4 is not liberated from catHDL at pH 5.6 and unbound PA at pH7.4 is captured by catHDL at pH5.6.

Next, we examined how much PA added at ratios of 0.03 and 2.4 interacted with catHDL (Fig. 2c, d). To do this, PA was labeled with a fluorescein derivative (see Experimental Sections). At $\mathrm{pH} 7.4$ at a ratio of 0.03 , PA was detected only at the same position as catHDL, while a significant amount of unbound PA (45\%) co-existed with catHDL/PA at a ratio of 2.4. It is unlikely that this unbound PA alone forms any micelles (or aggregates) comparable to catHDL/PA in terms of size (Table 1, Supplementary Fig.5). At pH 5.6, the unbound form of PA disappeared, and all PA co-localized with catHDL. These results show that catHDL can accommodate almost all PA added at a ratio of 0.03 at $\mathrm{pH} 7.4$, and that at $\mathrm{pH} 5.6, \mathrm{PA}$ in the complex may penetrate into the lipid bilayer of catHDL. In fact, low $\mathrm{pH}$-dependent complexation of poly(acrylic acid) derivatives with phospholipid bilayer membranes followed by solubilization of the latter was previously described, and may involve the formation of hydrogen bonds between the protonated carboxylate groups of the polymers and the phosphodiester functions of the lipid bilayer [25]. Thus, the ability of PA to penetrate into the nearby lipid bilayer could cause destabilization of catHDL structure and may also contribute to destabilizing the endosomal membrane [21].

Table 1 summarizes the size and zeta potential of catHDL at $\mathrm{pH} 7.4$ and 5.6 before and after the PA complexation. Data at $\mathrm{pH} 7.4$ and 5.6 were obtained for the complexes that were prepared at $\mathrm{pH} 7.4$, and then diluted by 10 -fold with a neutral or 
acidic buffer. The zeta potential of catHDL at $\mathrm{pH} 7.4$ was $14.1 \pm 2.8 \mathrm{mV}$, which was higher than $-11.2 \pm 1.4 \mathrm{mV}$ of the HDL mutant with no DOTAP, and it gradually decreased with increasing the amount of PA added. The effect of PA on the reduction of the zeta potential absolute value can be explained as follows. Firstly, at $\mathrm{pH} 7.4, \mathrm{PA}$ is negatively charged and can neutralize the positive charges of catHDL. Secondly, the PEG block of PA can create a hydrophilic shell, which would move away the slipping plane from the catHDL surface [15]. Upon acidification, the zeta potential of catHDL/PA at a PA/lipid ratio of 0.03 shifted from $0.2 \pm 0.3 \mathrm{mV}$ to $13.3 \pm 1.1 \mathrm{mV}$, which was almost the same as that of catHDL. The increase in zeta potential can be explained by the protonation of the MAA units and by the interactions of the PEG segment of PA with the protonated MAA block (via hydrogen bonds), which could result in the subsequent collapse of the PEG shell in the catHDL membrane [26]. At a higher PA/lipid ratio of 2.4, acidification of the medium brought back the zeta potential from $-11.6 \pm 2.0 \mathrm{mV}$ to only $-2.5 \pm 1.4 \mathrm{mV}$. These data were well in accordance with the agarose gel shift assay data (Fig. 2).

Table 1. Diameter and $\zeta$-potential of catHDL and catHDL/PA at different $\mathrm{pH}$

\begin{tabular}{ccccc}
\hline & \multicolumn{2}{c}{$\mathrm{pH} \mathrm{7.4}^{\text {a) }}$} & \multicolumn{2}{c}{$\mathrm{pH} \mathrm{5.6}^{\text {a) }}$} \\
\hline $\begin{array}{c}\text { PA/lipid } \\
\text { weight ratio }\end{array}$ & $\begin{array}{c}\text { Diameter } \\
{[\mathrm{nm}]}\end{array}$ & $\begin{array}{c}\zeta \text {-potential } \\
{[\mathrm{mV}]}\end{array}$ & $\begin{array}{c}\text { Diameter } \\
{[\mathrm{nm}]}\end{array}$ & $\begin{array}{c}\zeta \text {-potential } \\
{[\mathrm{mV}]}\end{array}$ \\
\hline 0 & $50.9 \pm 1.4$ & $14.1 \pm 2.8$ & $51.2 \pm 1.0$ & $14.7 \pm 3.9$ \\
0.03 & $51.3 \pm 0.5$ & $0.2 \pm 0.3$ & $53.0 \pm 0.8$ & $13.3 \pm 1.1$ \\
2.4 & $54.9 \pm 3.7$ & $-11.6 \pm 2.0$ & $58.4 \pm 3.1$ & $-2.5 \pm 1.4$
\end{tabular}

a) The value means $\mathrm{pH}$ at which the measurements were performed. After complexation at $\mathrm{pH}$ 7.4, catHDL/PA was diluted by 10-fold with 50 mM HEPES buffer (pH 7.4) or $50 \mathrm{mM}$ MES buffer ( $\mathrm{pH}$ 5.6). 


\subsection{Morphology of catHDL/PA complexes}

To investigate the morphology of catHDL and catHDL/PA, mica flake transmission electron microscopy (TEM) observation was performed (Fig. 3). Both catHDL and catHDL/PA had a broad size distribution at $\mathrm{pH} 7.4$, and neither complexation with PA nor lowering $\mathrm{pH}$ significantly affected the morphology. In all the images, a few discoidal structures of $50-80 \mathrm{~nm}$ in diameter, which were comparable to the dynamic light scattering (DLS) data (Table 1), were observed, but 15-20 nm particles mainly existed. In our previous study, a similar apparent discrepancy in size between DLS and TEM analyses were observed, although to a lesser extent than in this work $[13,14]$. Therefore, catHDL may consist of relatively loose aggregates of smaller nanoparticles in solution and disaggregation might somehow occur during procedure for mica flake TEM analysis. In fact, faint joint lines in the $50-80 \mathrm{~nm}$ discoidal structure were observed especially in Fig. 3d. We analyzed catHDL and the parent HDL mutant (mean size of $15 \mathrm{~nm}$ by DLS analysis) by size exclusion chromatography (Supplementary Fig. 6). In both cases, single peaks appeared, but the retention time was different, $15.039 \mathrm{~min}$ for 50.9-nm catHDL and $15.644 \mathrm{~min}$ for 15.0-nm parent HDL mutant. This shorter retention time for catHDL would support its larger size in solution than $15 \mathrm{~nm}$. Further studies should be performed to address this structural heterogeneity of catHDL. 

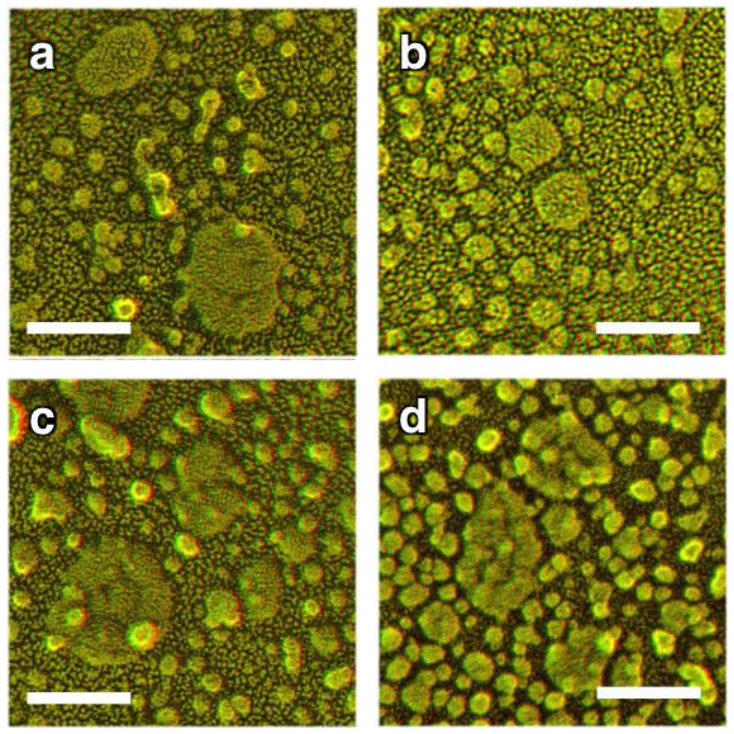

Fig. 3. Morphology of catHDL/PA complex. Mica flake transmission electron microscopy images of catHDL (a, c) and catHDL/PA complex with PA/lipid wieght ratio of 0.03 (b, d) were observed at pH $7.4(\mathrm{a}, \mathrm{b})$ and pH 5.6 (c, d). In any cases, discoidal structure with a broad size distribution is observed. Discoidal structure of catHDL is not affected by complexation with PA and pH conditions. Scale Bar, $50 \mathrm{~nm}$.

\subsection{Intracellular trafficking of catHDL/PA complex}

In order to analyze the intracellular trafficking of catHDL/PA, prepared at a PA/lipid weight ratios of 0.03 and 2.4 , for longer periods $(>1 \mathrm{~h})$, the cells were treated with the tetramethylrhodamine isothiocyanate (TRITC)-labeled complexes in the presence of serum (Fig. 4). The treatment period was prolonged to facilitate the assessment of the effect of PA on intracellular trafficking by allowing more catHDL/PA to be internalized because internalization of catHDL/PA was significantly low for $1 \mathrm{~h}$ at neutral $\mathrm{pH}$ in the absence of serum, compared with catHDL (Fig. 1). It was first verified that catHDL/PA was stable even in the presence of $10 \%$ serum (Supplementary Fig. 7). The late endosomes/lysosomes were labeled with LysoTracker Green. After $1 \mathrm{~h}$ of 
treatment with the TRITC-labeled catHDLs without PA, the majority of the TRITC fluorescence (50-60\%) was associated to the late endosomes/lysosomes as indicated by the colocalization ratio (see Experimetal Sections) (Fig. 4a, d). After over $3 \mathrm{~h}$ of incubation, the TRITC fluorescence outside the endo/lysosomal organelles was slightly increased, and thus the colocalization ratio decreased to less than $40 \%$ (Fig. 4a, d). In the presence of PA, however, the intracellular location and dynamics showed different profiles. The TRITC fluorescence was diffusively distributed in the cytoplasm even after $1 \mathrm{~h}$, to yield the colocalization ratio of less than $25 \%$ (Fig. $4 \mathrm{~b}-\mathrm{d}$ ). The ratios at an earlier time point (30 min) were almost the same as those at $1 \mathrm{~h}$ (Supplementary Fig. 8). Longer treatment also appeared to have a marginal effect on the colocalization ratio, while persistently increasing internalization of catHDLs (Supplementary Fig. 9). Upon co-treatment with an endosomal acidification inhibitor, bafilomycin A1, the ratio for catHDL/PA significantly increased (Supplementary Fig. 10). These results demonstrate that PA in the complexes is able to enhance the endosomal escape of, at least, the protein component of catHDL within $1 \mathrm{~h}$ of treatment and that the protonation of PA in the endosomes, which induces its coil-to-globule transition that can destabilize the endosomal membrane [7], is also responsible for the endosomal escape phenomena observed here. The effects of PA on internalization and endosomal escape of catHDL were summarized in Fig. 5. 

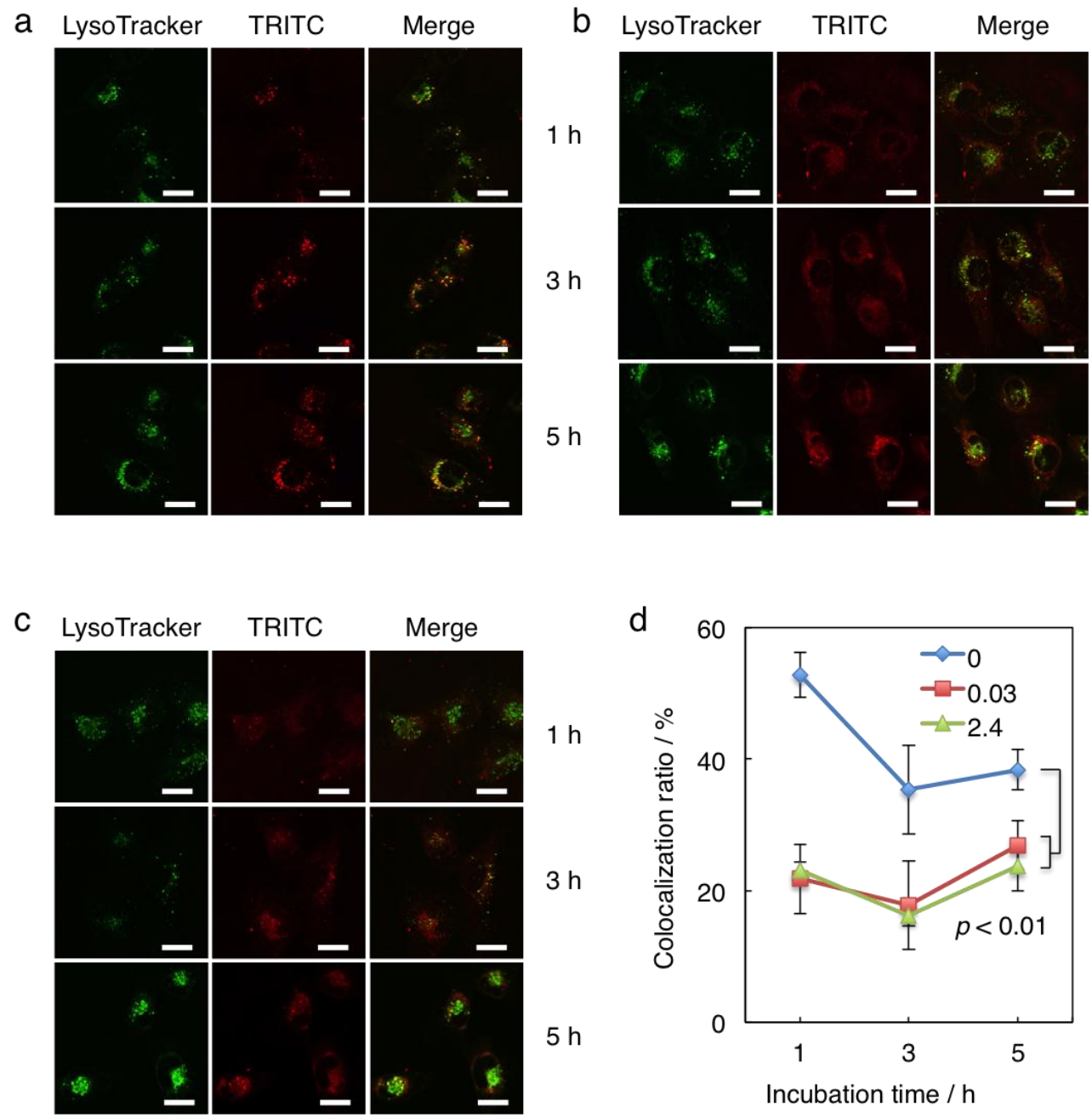

Fig. 4. Improved endosomal escape of catHDL component by complexation with PA. Time-dependent confocal analysis of the cells treated with (a) catHDL, and catHDL/PA at a PA/lipid weight ratio of (b) 0,03 or (c) 2.4. All experiments were performed in McCoy's 5A medium in the presence of $10 \%$ FBS at pH 7.4. catHDL was labeled with TRITC (red), and the late endosomes/lysosomes were labeled with LysoTracker Green (green). For catHDL/PA, TRITC fluorescence is prominently detected outside the endosomes/lysosomes even after $1 \mathrm{~h}$, indicating improvement of the endosomal escape at least of the protein component of catHDL by PA. Scale bar, $10 \mu \mathrm{m}$. (d) Time-dependent change in the ratio of colocalization between TRITC and LysoTracker Green fluorescences (see Materials and methods Section). PA complexation significantly decreases the colocalization ratio even after $1 \mathrm{~h}$ of the treatment, and it is almost unchanged up to $5 \mathrm{~h}$ although catHDL/PA compexes persistently internalized. 
TRITC fluorescence signal in (b) and (c) was intensified for clarification in the same manner. The same intensification parameter was used for all images for catHDL/PAs because it affected the colocalization ratio. Time-dependent change in the total number of red pixels, used for computing this ratio, was shown in supplementary Fig. 9. As a result of intensification, their total number became comparable between catHDL and catHDL/PAs. Numbers in the graph indicate a PA/lipid weight ratio. The $p$ value was evaluated by one-way ANOVA.

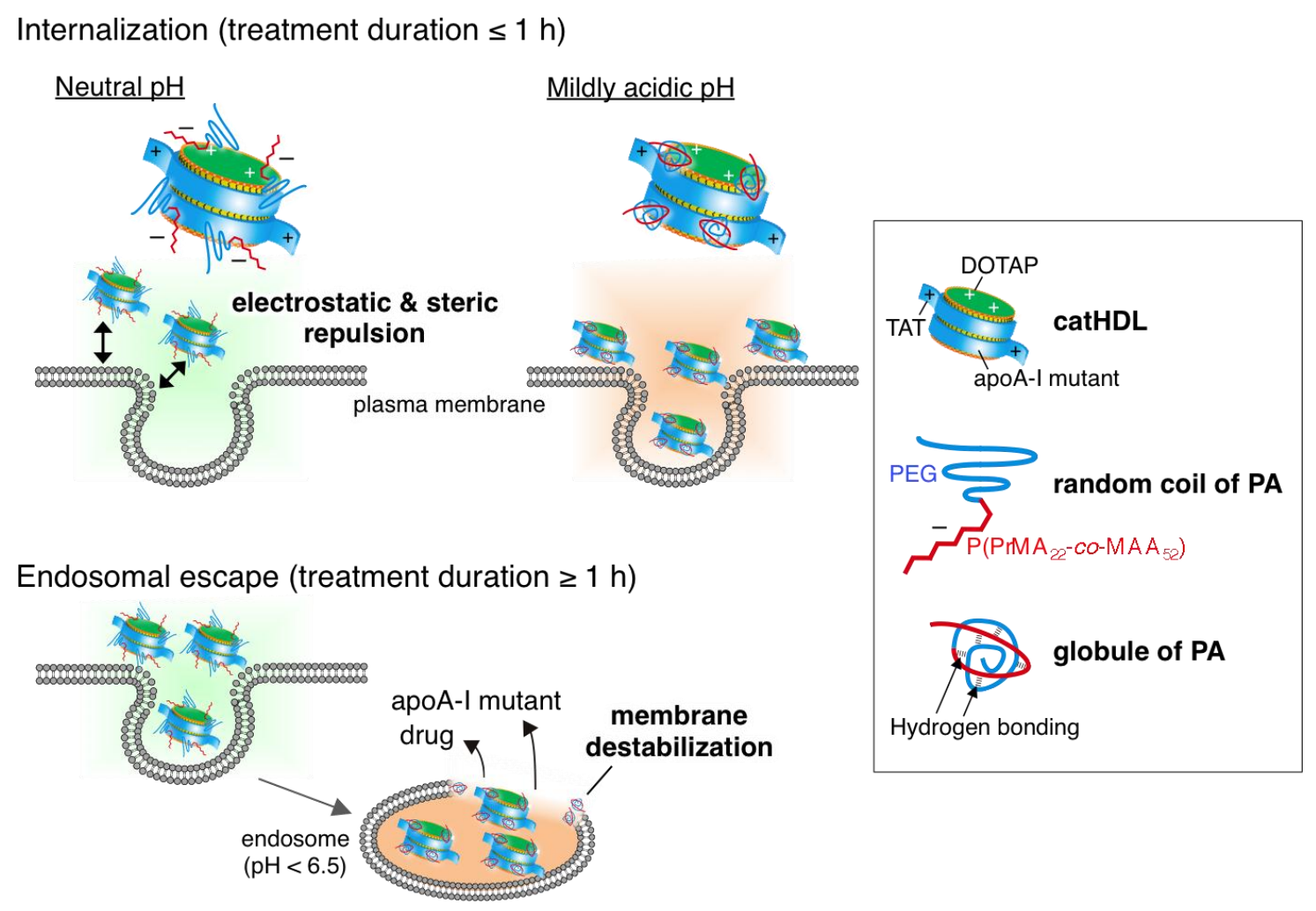

Fig. 5. Schematic illustration of $\mathrm{pH}$-dependent internalization and endosomal escape of catHDL/PA. At neutral $\mathrm{pH}$, internalization of catHDL/PA is suppressed most likely due to electrostatic and steric repulstion induced by PA in the random coil state. At mildly acidic $\mathrm{pH}, \mathrm{PA}$ bound to catHDL changes their conformation from a random coil to a hydrophobic globule through the protonation of the carboxylate groups followed by hydrogen bonding between the protonated groups and the polyethylene glycol (PEG) segment of PA and penetrates into the hydrophobic core of catHDL, leading to the 
recovery of catHDL internalization. Upon internalization of catHDL/PA at neutral $\mathrm{pH}$, albeit at a slower rate, the mildly acidic condition in the endo/lysomes also induces the conformational change of PA bound to catHDL, which could destabilize the endo/lysosomal membranes to allow the escape of the protein component of catHDL and the drugs in catHDL outside the endo/lysosomes.

\subsection{Intracellular delivery of anticancer drugs by catHDL/PA complex}

As described above, DXR encapsulated into the HDL mutant (with TAT peptide, without DOTAP) was delivered into the endosomes after $1 \mathrm{~h}$ of treatment, and only a small portion of DXR in the endosomes was escaped from there after the following 24-h culture, while the antitumor activity was successfully enhanced, compared with that of the TAT-lacking complex. Here, catHDL was mixed with DXR at $50^{\circ} \mathrm{C}$ for $60 \mathrm{~min}$ [18] prior to complexation with PA in order to examine intracellular localization of the cargo delivered by catHDL/PA. The DXR loading efficiency for catHDL (1.5\%) was comparable to that for the HDL mutant (1.4\%) and their in vitro DXR release rate was also similar (Supplementary Fig. 11), indicating that the presence of DOTAP does not affect the DXR loading and release. The mean diameters were similar among DXR-loaded catHDL (DXR@ @atHDL, $46.9 \pm 0.9 \mathrm{~nm}$ ) and DXR-loaded catHDL/PA (DXR@ @ catHDL/PA) at a PA/lipid ratio of $0.03(48.9 \pm 0.8 \mathrm{~nm})$ and of 2.4 $(57.1 \pm 2.3 \mathrm{~nm})$. After $18 \mathrm{~h}$ of treatment of the cells with these DXR formulations in $10 \%$ serum, DXR fluorescence was observed mainly in the nucleus, which is its major site of action (Fig. 6a), regardless of the presence or absence of PA (Fig. 6b-d). Even after $5 \mathrm{~h}$ of treatment, nuclear delivery of DXR by catHDL and catHDL/PA was obvious, and the colocalization of DXR with the endo/lysosomal organelles decreased from $49 \%$ to $34 \%$ 
by complexation with PA (supplementary Fig. 12). After $18 \mathrm{~h}$ of incubation, the colocalization ratio further decreased from $10 \%$ to $5 \%$ following complexation with PA at the two PA/lipid ratios (Fig. 6e). This result clearly shows that although not so drastically as displayed in Fig. 4, the endosomal escape of the cargo of catHDL was also enhanced by the PA complexation. Meanwhile, the high nuclear DXR delivery efficiency of catHDL, irrespective of the presence or absence of PA, was unexpected because our previous study using DXR-loaded HDL mutant (no DOTAP) showed its predominant localization in the endosomes and its in vitro DXR release rate only slightly slower than that shown in Supplementary Fig. 11. The mechanism of the increased nuclear delivery is unclear at present, but the cationic surface of DXR@catHDL $(14.1 \pm 2.8 \mathrm{mV})$ may play a role in destabilization of endosomal membrane [27]. In any case, given that the site of action of DXR is the nucleus, this intrinsic property of catHDL could be advantageous.

The effect of the PA complexation on the cell uptake and cytotoxicity of DXR was quantitatively investigated. After 18-h treatment of the cells, the uptake of DXR was reduced in the presence of PA (Fig. 6f), which was in agreement with the results in Fig. 1. The degree of reduction was dependent on the amount of PA added. This dependency would be accounted for by decrease in electrostatic interaction between catHDL/PA and the cell [28]. On the other hand, the three DXR formulations showed comparable cytotoxicity, while no obvious cytotoxicity was observed for empty catHDL and catHDL/PA (Fig. 6g). Therefore, we hypothesized that this comparable cytotoxicity of the DXR formulations was a result of compensation of the decreased DXR uptake by the increased endosomal escape. 

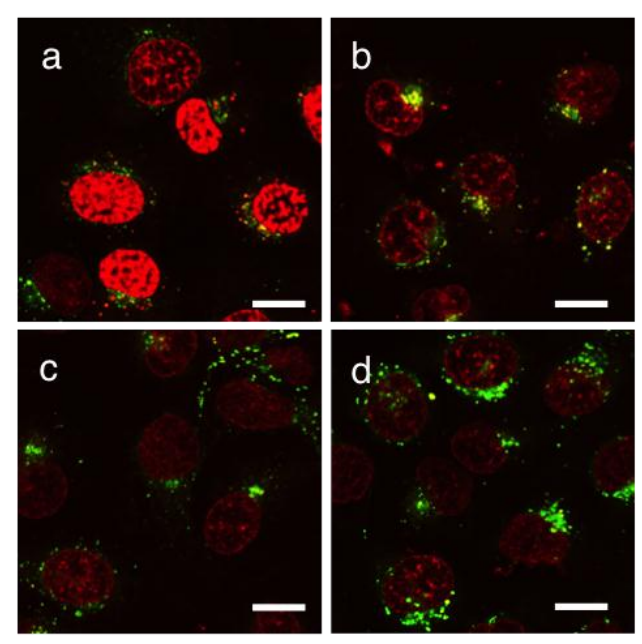

$f$

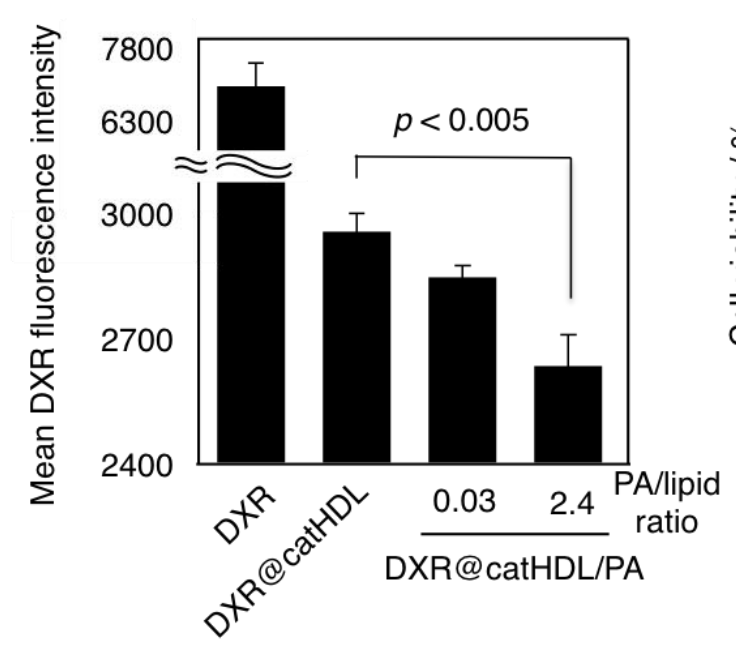

e
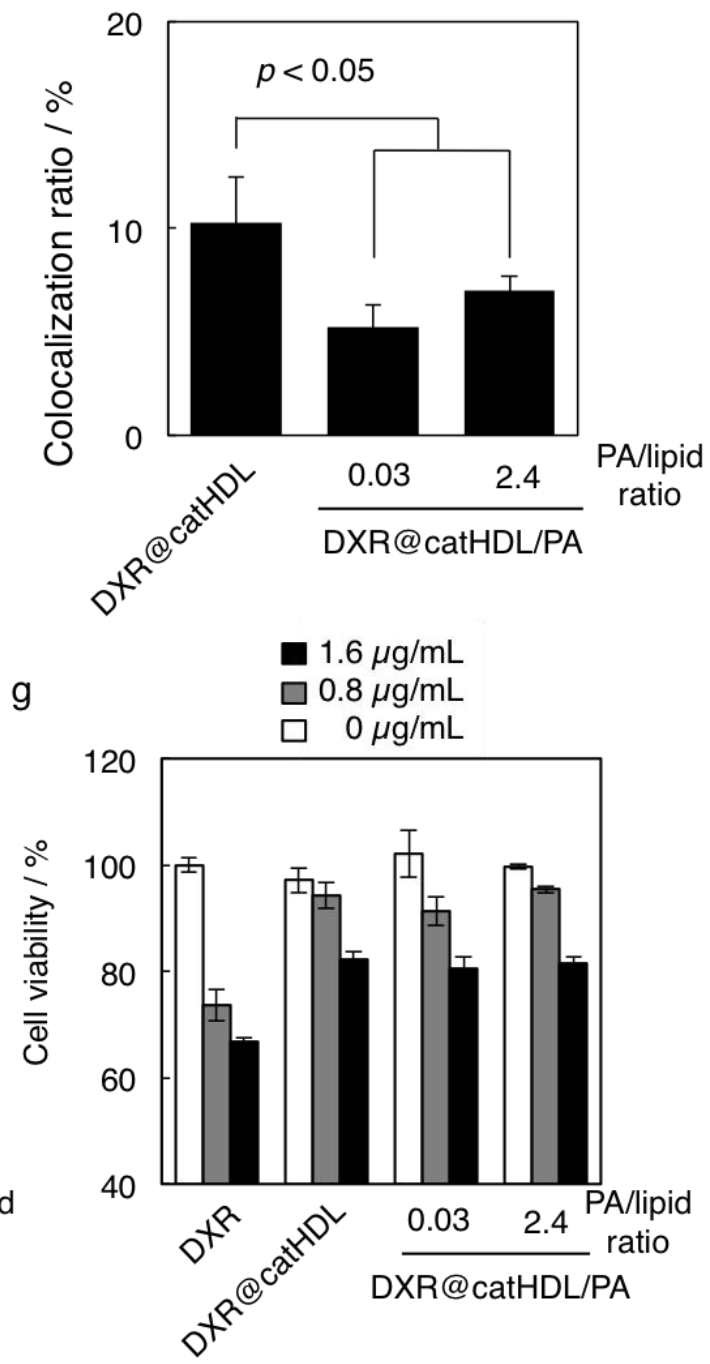

Fig. 6. Improved endosomal escape of anticancer drug in catHDL/PA. Doxorubicin (DXR)-loaded catHDL/PA (DXR@ @ catHDL/PA) was prepared by incubation of catHDL with DXR at $50^{\circ} \mathrm{C}$ followed by with PA at room temperature at $\mathrm{pH} 7.4$ (see Materials and methods Section). All data were for the cells treated with the DXR formulations for $18 \mathrm{~h}$ in McCoy's 5A medium in the presence of 10\% FBS at $\mathrm{pH}$ 7.4. Confocal images of the cells were acquired for (a) free DXR, (b) DXR@catHDL, and DXR@catHDL/PA at a PA/lipid ratio of (c) 0.03 and (d) 2.4 (3.2 $\mu \mathrm{g} / \mathrm{mL}$ DXR). The merged images of DXR (red) and LysoTracker Green fluorescences (green) were shown. For clarity, DXR fluorescence signals in (b)-(d) were intensified in the same manner. While DXR fluorescence is observed mainly in the nucleus, regardless of the presence or absence of 
PA, colocalization of the red and green fluorescence signals appears to be decreased in the presence of PA. Scale bar, $10 \mu \mathrm{m}$. (e) Colocalization ratios of DXR and LysoTracker Green fluorescences (see Materials and methods Section). The lower ratios for DXR@catHDL/PA than for DXR@catHDL indicate that endosomal escape of DXR is also enhanced by the PA complexation. This effect of PA for DXR was already obvious after $5 \mathrm{~h}$ of treatment (supplementary Fig. 12). (f) Fluorescence-activated cell sorter (FACS) analysis by using DXR fluorescence. The 3,000 cells treated at 1.6 $\mu \mathrm{g} / \mathrm{mL}$ on a DXR basis were counted in each condition $(\mathrm{n}=3)$. The uptake of DXR is reduced in the presence of PA, which is in accordance with the data in Fig. 1. (g) Cell viability after treatment at $0,0.8$ and $1.6 \mu \mathrm{g} / \mathrm{mL}$ DXR $(\mathrm{n}=3)$. Although PA reduces the uptake of DXR in catHDL, its cytotoxic effect is comparable between DXR@catHDL/PA and DXR@catHDL, indicating that PA possibly increases the efficacy of DXR taken up by the cells. The $p$ value was evaluated by one-way ANOVA.

Whether the catHDL/PA could be applied to another anticancer drug, we encapsulated another green fluorescent anticancer compound, curcumin (CCM) into the catHDL $[29,30]$. The diameter of CCM-loaded catHDL (CCM@catHDL) and CCM-loaded catHDL/PA (ratio 2.4, CCM@ catHDL/PA) was $43.2 \pm 0.5$ and $45.0 \pm 1.2$ $\mathrm{nm}$. After $5 \mathrm{~h}$ of treatment of the cells with the two CCM formulations, the fluorescence was observed mainly from the cytoplasm, regardless of the presence or absence of PA (supplementary Fig. 13a-c). Unlike in the case of DXR, the effect of the PA complexation on the endosomal escape of CCM was less obvious (supplementary Fig. 13d), but the cell uptake of CCM was significantly reduced in the presence of PA (supplementary Fig. 13e). The mean CCM fluorescence intensity was 4270 for CCM@catHDLvs. 2043 for CCM@catHDL/PA, which corresponded to the values for the cells treated with 12.2 and $6.8 \mu \mathrm{g} / \mathrm{mL}$ free CCM, respectively, based on a standard curve of mean CCM fluorescence intensity against free CCM concentration at which the 
cells were treated (supplementary Fig. 13f). When cytotoxicity of CCM@catHDL and CCM@catHDL/PA was compared with that of 12.2 and $6.8 \mu \mathrm{g} / \mathrm{mL}$ free CCM, there was no significant difference between CCM@ catHDL and free CCM, but a more potent cytotoxicity was observed for CCM@ catHDL/PA than free CCM (supplementary Fig. 13g). Although the endosomal escape of CCM induced by PA was less obvious, these results obtained with the two drugs suggest that PA is capable of potentiating the pharmacological action of the cargoes delivered by catHDL in the cells through their endosomal escape.

There are relatively limited number of reports on modification of pre-formed cationic nanoparticles with polyanions for design of membranolytic nanoparticles [21, 31, 32], while simultaneous mixing of polyanions, cationic molecules and cargoes have been widely adopted for their preparation. Kiang et al. demonstrated that the transfection efficiency of complexes prepared by mixing polyanions and chitosan/plasmid DNA nanoparticles was superior to that obtained by mixing the three components at the same time [31]. In this study, we successfully prepared stable catHDL/PA complexes even in the presence of $10 \%$ serum by mixing catHDL and PA, and demonstrated for the first time that catHDL/PA was better internalized by the cells at low $\mathrm{pH}$ and could enhance endosomal escape of the cargoes. On the other hand, catHDL/PA did not increase the pharmacological action of the cargo compared with catHDL, whereas the transfection efficiency of the above-mentioned chitosan/plasmid DNA nanoparticles was increased upon addition of polyanions [31]. This would be due to the significant attenuation of internalization of catHDL/PA at neutral $\mathrm{pH}$ (Fig. 1), which could diminish the beneficial effect of its membranolytic activity. This consideration was supported by the observation that once CCM@catHDL/PA was 
internalized, PA was able to potentiate CCM activity in the cells (supplementary Fig. 13g). This issue could be solved by derivatizing the PEG block of PA with a targeting ligand in order to promote cell uptake $[15,16]$. On the other hand, Peddada et al. reported that a polyanion grafted with poly(oxyalkylene amines) enhanced internalization of the DOTAP/oligo DNA/polyanion complex, resulting in an increase in in vitro transfection efficiency [32]. However, upon this grafting, the $\mathrm{pH}$-dependent membranolytic functionality of the parent polyanion was lost. Thus, the significance of the $\mathrm{pH}$-dependent internalization ability of our catHDL should be assessed via in vivo studies in the future.

\section{Conclusions}

In this study, HDL was cationized both genetically and chemically for the first time with the cationic peptide (TAT) and cationic lipids (DOTAP or DC-chol), respectively. Following the complexation with the anionic block copolymer (PA), the cationized HDL mutant prepared with TAT and 10 mol\% DOTAP (catHDL) showed a low $\mathrm{pH}$-dependent internalization in a clearest manner. The PA binding to catHDL was saturable and stable at $\mathrm{pH} 7.4$ even in the presence of serum. The TEM structure of catHDL was discoidal like the parent HDL mutant, and it remained essentially unchanged at $\mathrm{pH} 7.4$ and 5.6 in the presence of PA. Improved endosomal escape of the protein components and drugs in catHDL was demonstrated in the presence of PA after $1 \mathrm{~h}$ of incubation with human cancer cells. More importantly, catHDL/PA was able to potentiate the cytotoxicity of the drugs taken up by the cells. This is the first demonstration of $\mathrm{pH}$-dependent control of internalization and endosomal escape of 
HDL cargoes. The efficacy of the anticancer drug-loaded catHDL/PA systemically administerd could be further enhanced in tumor tissues being in mildly acidic $\mathrm{pH}$ or through functionalization with targeting ligands.

\section{Acknowledgements}

This work was supported by JSPS KAKENHI Grand Number 26870301 (H.K.) and the World Premier International Research Center Initiative (WPI), MEXT, Japan. JCL acknowledges funding from the Swiss National Science Foundation (grant 310030_138342).

\section{References}

[1] D.J. Klionsky, S.D. Emr, Autophagy as a regulated pathway of cellular degradation, Science 290 (2000) 1717-1721.

[2] W.A. Dunn, Autophagy and related mechanisms of lysosome-mediated protein degradation, Trends Cell Biol. 4 (1994) 139-143.

[3] R. Sakhtianchi, R.F. Minchin, K.B. Lee, A.M. Alkilany, V. Serpooshan, M. Mahmoudi, Exocytosis of nanoparticles from cells: role in cellular retention and toxicity, Adv. Colloid Interface Sci. 201-202 (2013) 18-29.

[4] A.K. Varkouhi, M. Scholte, G. Storm, H.J. Haisma, Endosomal escape pathways for delivery of biologicals, J. Control. Release 151 (2011) 220-228.

[5] F.R. Maxfield, T.E. McGraw, Endocytic recycling, Nat. Rev. Mol. Cell Biol. 5 (2004) 121-132. 
[6] W. Gao, J.M. Chan, O.C. Farokhzad, pH-responsive nanoparticles for drug delivery, Mol. Pharm. 7 (2010) 1913-1920.

[7] M.-A. Yessine, J.-C. Leroux, Membrane-destabilizing polyanions: interaction with lipid bilayers and endosomal escape of biomacromolecules, Adv. Drug Deliv. Rev. 56 (2004) 999-1021.

[8] D.W. Pack, A.S. Hoffman, S. Pun, P.S. Stayton, Design and development of polymers for gene delivery, Nat. Rev. Drug Discov. 4 (2005) 581-593.

[9] L.E. Gerweck, K. Seetharaman, Cellular $\mathrm{pH}$ gradient in tumor versus normal tissue : potential exploitation for the treatment of cancer, Cancer Res. 56 (1996) 1194-1198.

[10] J.D. Glickson, S. Lund-Katz, R. Zhou, H. Choi, I. Chen, H. Li, I. Corbin, A.V. Popov, W. Cao, L. Song, C. Qi, D. Marotta, D.S. Nelson, J. Chen, B. Chance, G. Zheng, Lipoprotein nanoplatform for targeted delivery of diagnostic and therapeutic agents, Mol. Imaging 7 (2008) 101-110.

[11] P.C. Rensen, R.L. Vrueh, J. Kuiper, M.K. Bijsterbosch, E.A. Biessen, T.J. Berkel, Recombinant Lipoproteins : lipoprotein-like lipid particles for drug targeting, Adv. Drug Deliv. Rev. 47 (2001) 251-276.

[12] T. Murakami, W. Wijagkanalan, M. Hashida, K. Tsuchida, Intracellular drug delivery by genetically engineered high-density lipoprotein nanoparticles, Nanomedicine (Lond) 5 (2010) 867-879.

[13] S. Mathew, T. Murakami, H. Nakatsuji, H. Okamoto, N. Morone, J.E. Heuser, M. Hashida, H. Imahori, Exclusive photothermal heat generation by a gadolinium bis(naphthalocyanine) complex and inclusion into modified 
high-density lipoprotein nanocarriers for therapeutic applications, ACS Nano 7 (2013) 8908-8916.

[14] T. Numata, T. Murakami, F. Kawashima, N. Morone, J.E. Heuser, Y. Takano, K. Ohkubo, S. Fukuzumi, Y. Mori, H. Imahori, Utilization of photoinduced charge-separated state of donor-acceptor-linked molecules for regulation of cell membrane potential and ion transport, J. Am. Chem. Soc. 134 (2012) 60926095

[15] M. Elsabahy, N. Wazen, N. Bayó-Puxan, G. Deleavey, M. Servant, M.J. Damha, J.-C. Leroux, Delivery of nucleic acids through the controlled disassembly of multifunctional nanocomplexes, Adv. Funct. Mater. 19 (2009) 3862-3867.

[16] A.E. Felber, B. Castagner, M. Elsabahy, G.F. Deleavey, M.J. Damha, J.-C. Leroux, siRNA nanocarriers based on methacrylic acid copolymers, J. Control. Release 152 (2011) 159-167.

[17] D.P. Rogers, C.G. Brouillette, J.A. Engler, S.W. Tendian, L. Roberts, V.K. Mishra, G.M. Anantharamaiah, S. Lund-Katz, M.C. Phillips, M.J. Ray, Truncation of the amino terminus of human apolipoprotein A-I substantially alters only the lipid-free conformation, Biochemistry 36 (1997) 288-300.

[18] T. Murakami, K. Tsuchida, M. Hashida, H. Imahori, Size control of lipid-based drug carrier by drug loading, Mol. BioSyst. 6 (2010) 789-791.

[19] K. Takata, S. Horiuchi, A.T. Rahim, Y. Morino, Receptor-mediated internalization of high density lipoprotein by rat sinusoidal liver cells : identification of a nonlysosomal endocytic pathway by fluorescence-labeled ligand, J. Lipid Res. 29 (1988) 1117-1126. 
[20] S. Takae, K. Miyata, M. Oba, T. Ishii, N. Nishiyama, K. Itaka, Y. Yamasaki, H. Koyama, K. Kataoka, PEG-detachable polyplex micelles based on disulfide-linked block catiomers as bioresponsive nonviral gene vectors, J. Am. Chem. Soc. 130 (2008) 6001-6009.

[21] M.-A. Yessine, C. Meier, H.-U. Petereit, J.-C. Leroux, On the role of methacrylic acid copolymers in the intracellular delivery of antisense oligonucleotides, Eur. J. Pharm. Biopharm. 63 (2006) 1-10.

[22] G. Meer, D.R. Voelker, G.W. Feigenson, Membrane lipids: where they are and how they behave, Nat. Rev. Mol. Cell Biol. 9 (2008) 112-124.

[23] N.J. Zuidam, Y. Barenholz, Electrostatic parameters of cationic liposomes commonly used for gene delivery as determined by 4-heptadecyl-7-hydroxycoumarin, Biochim. Biophys. Acta 1329 (1997) 211222.

[24] R. Kim, Native agarose gel electrophoresis of multiprotein complexes, Cold Spring Harb. Protoc. 7 (2011) 884-887.

[25] K. Seki, D.A. Tirrell, pH-dependent complexation of poly(acrylic acid) derivatives with phospholipid vesicle membrane, Macromolecules 17 (1984) $1692-1698$.

[26] P. Satturwar, M.N. Eddine, F. Ravenelle, J.-C. Leroux, pH-responsive polymeric micelles of poly(ethylene glycol)-b-poly(alkyl(meth)acrylate-co-methacrylic acid): influence of the copolymer composition on self-assembling properties and release of candesartan cilexetil, Eur. J. Pharm. Biopharm. 65 (2007) 379-387. 
[27] Y. Xu, F. C. Szoka, Jr., Mechanism of DNA release from cationic liposome/DNA complexes used in cell transfection, Biochemistry, 35 (1996) $5616-5623$.

[28] C. He, Y. Hu, L. Yin, C. Tang, C. Yin, Effects of particle size and surface charge on cellular uptake and biodistribution of polymeric nanoparticles, Biomaterials 31 (2010) 3657-3666.

[29] T. Esatbeyoglu, P. Huebbe, I.M. Ernst, D. Chin, A.E. Wagner, G. Rimbach, Curcumin-from molecule to biological function, Angew. Chem. Int. Ed. 51 (2012) 5308-5332.

[30] T. Harada, L. Giorgio, T.J. Harris, D.-T. Pham, H.T. Ngo, E.F. Need, B.J. Coventry, S.F. Lincoln, C.J. Easton, G. Buchanan, T.W. Kee, Diamide linked gamma-cyclodextrin dimers as molecular-scale delivery systems for the medicinal pigment curcumin to prostate cancer cells, Mol. Pharm. 10 (2013) $4481-4490$.

[31] T. Kiang, C. Bright, C.Y. Cheung, P.S. Stayton, A.S. Hoffman, K.W. Leong, Formulation of chitosan-DNA nanoparticles with poly(propyl acrylic acid) enhances gene expression, J. Biomater. Sci. Polym. Ed. 15 (2004) 1405-1421.

[32] L.Y. Peddada, N.K. Harris, D.I. Devore, C.M. Roth, Novel graft copolymers enhance in vitro delivery of antisense oligonucleotides in the presence of serum, J. Control. Release 140 (2009) 134-140. 


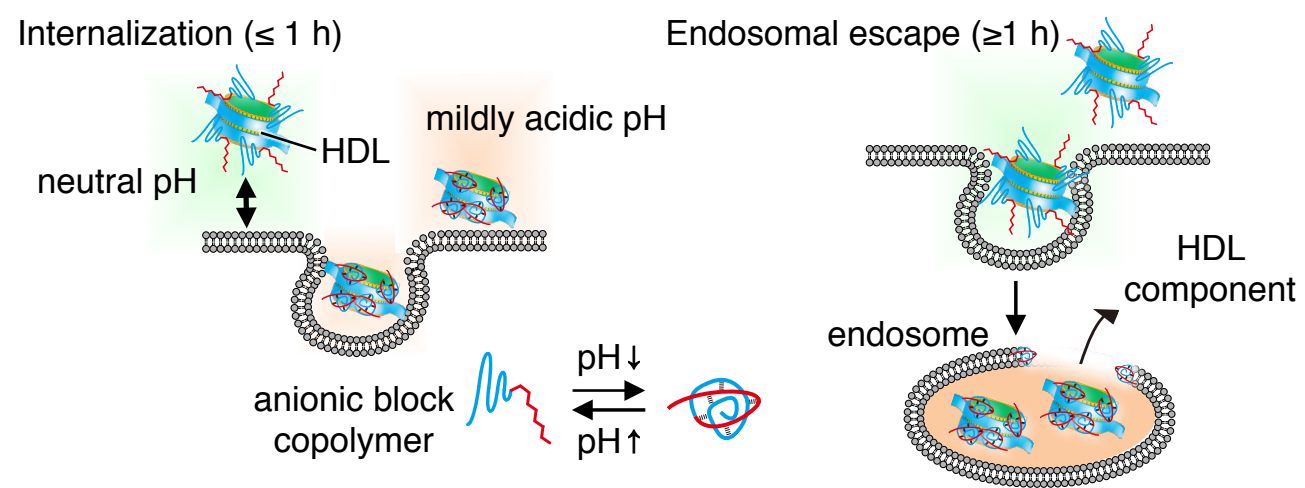

